\title{
Preparation and Characterization of Three-Dimensional Pt Nanoparticle Networks
}

\author{
H. Bönnemann* and N. Waldöfner
}

Max-Planck-Institut für Kohlenforschung, D-45466 Mülheim an der Ruhr, Germany

H.-G. Haubold and T. Vad

Institut für Festkörperforschung, Forschungszentrum J ülich GmbH, D-52425 J ülich, Germany

Received J uly 19, 2001. Revised Manuscript Received November 2, 2001

We have synthesized nanostructured metal/organic networks by cross-linking preformed aluminum-organic-stabilized Pt nanoparticles in solution with organic spacer molecules. The protonolytic cross-linking mechanism presented makes use of reactive aluminum methyl groups at the surface of the particles. The particles and their networks have been characterized by el ectron microscopy and IR spectroscopy. For the structural characterization of the hybrids, anomalous small-angle X-ray scattering (ASAXS) was applied.

\section{Introduction}

The assembly of nanosized metal particles into ordered arrays has become a new trend in chemistry over the past few years. This topic is part of the rapidly evolving field of chemical nanotechnology, which is believed to be one of the key technologies of the near future. The effort is mainly driven by the idea of creating new nanostructured materials in a bottom-up approach, starting from simple components such as colloids or even molecules. For example, the integration of nanoscaled metal particles into electronic or optical devices is interesting because of quantum size effects that a single particle exhibits when the number of atoms is reduced to a few hundred or less. 1,2 Moreover, the new resulting materials are expected to exhibit new collective properties that differ from those of the bulk materials. In all of these fields, the uniformity of nanoparticle size and arrangement plays a crucial role in controlling the properties of these materials. Nanocrystal superlattices and colloidal networks have been prepared by several groups. ${ }^{3-15}$ However, bottom-up preparations of nanoparticles to give three-dimensi onal structures still

(1) Schön, G. Simon, U Colloid Polym Sci. 1995, 273, 101-117.

(2) Schön, G.; Simon, U. Colloid Polym. Sci. 1995, 273, 202-218.

(3) Park, S. J .; Lazarides, A. A.; Mirkin, C. A. Angew. Chem., Int. Ed. 2000, 39, 3845-3848.

(4) Reetz, M. T.; Winter, M.; Tesche, B. Chem. Commun. 1997, 147148.

(5) Sato, T.; Brown, D.; J ohnson, B. F. G. Chem. Commun. 1997, 1007-1008.

(6) Schmid, G.; Bäumle, M.; Beyer, N. Angew. Chem., Int. Ed. 2000, 39, 182-184.

(7) Taleb, A.; Petit, C.; Pileni, M. P. Chem. Mater. 1997, 9, 950959.

(8) Motte, L.; Billoudet, F.; Lacaze, E.; Doulin, J .; Pileni, M. P. J Phys. Chem. B 1997, 101, 138-144.

(9) Lin, X. M.; Sorensen, C. M.; Klabunde, K.J . Chem. Mater. 1999, $11,198-202$.

(10) Brust, M.; Bethell, D.; Schiffrin, D. J .; Kiely, C. J . Adv. Mater. 1995, 7 (9), 795-797.

(11) Shenton, W.; Davis, S. A.; Mann, S. Adv. Mater 1999, 11 (6), 449-452. 1205. remain a challenge for synthetic chemists. Here, we report a new preparation pathway and the characterization of metal/organic networks formed by the crosslinking of aluminum-organic-stabilized platinum nanoparticles with bifunctional spacer molecules.

The reductive stabilization of nanoparticles with aluminum trialkyls is a preparation method that was recently devel oped by Bönnemann and co-workers. ${ }^{16,17}$ This method has been shown to be a reliable approach for the production of a wide range of small zero-valent transition metal particles that can be redispersed in organic solvents such as toluene or THF. The key feature of this synthesis is the formation of an organometallic colloidal protecting shell around the particles. Highly reactive aluminum alkyl groups present in the shell open up the possibility for chemical reactions at the surface of a colloidal particle. One example is the substitution of organic groups at the aluminum to modify the dispersive properties of the colloids. ${ }^{17}$ The substituents can also be bifunctional, in which case, a cross-linking of the particles can be brought about that leads to the formation of a 3-D nanoparticle network. In this network, control of the interparticle distance can be achieved by varying the length of the spacer molecules.

In addition to applying el ectron microscopy, we investigated the physical properties of the Al organic shell with IR spectroscopy. For the structural characterization of the colloids, anomalous small-angle X-ray scat-

(13) Korgel, B. A.; Fullam, S.; Connolly, S.; Fitzmaurice, D. J . Phys. Chem. B 1998, 102, 8379-8388.

(14) Park, S. J .; Lazarides, A. A.; Mirkin, C. A.; Brazis, P. W.; Rannewurf, C. R.; Letzinger, R. L. Angew. Chem., Int. Ed. 2000, 39 (21), 3845-3848

(15) Simon, U.; Flesch, R.; Wiggers, H.; Schön, G.; Schmid, G. J . Mater. Chem. 1998, 8 (3), 517-518.

(16) Bönnemann, H.; Brijoux, W.; Brinkmann, R.; Endruschat, U.; Hofstadt, W.; Angermund, K. Rev. Roum. Chim. 1999, 11-12, 10031010.

(17) Bönnemann, H.; Brijoux, W.; Brinkmann, R. (Studiengesellschaft Kohle mbH, DE). German Patent DE 19821968 A, 1999. 


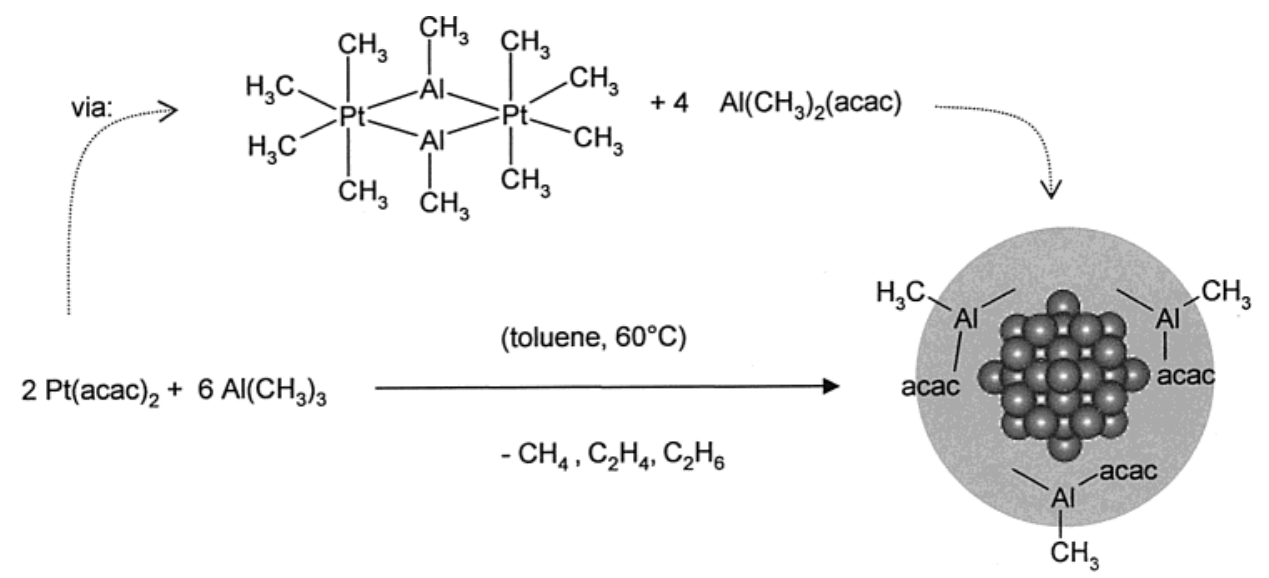

Figure 1. Schematic representation of the synthesis of aluminum-organic-stabilized Pt nanoparticles (acac $=$ acetylacetonate).

tering (ASAXS) was applied. This method provides information about particle size and arrangement in the colloid. Furthermore, it opens up the possibility of separating the scattering of the particles from that of the surrounding organic components.

\section{Experimental Section}

Synthesis of Aluminum-Organic-Stabilized Pt ColIoids. Platinum(II) acetylacetonate $(1.97 \mathrm{~g}, 5 \mathrm{mmol})$ was dissolved under argon atmosphere in $200 \mathrm{~mL}$ of dry toluene. $\mathrm{Al}\left(\mathrm{CH}_{3}\right)_{3}(1.44 \mathrm{~g}, 20 \mathrm{mmol})$ was dissol ved in $200 \mathrm{~mL}$ of toluene and carefully added over $4 \mathrm{~h}$ at $60^{\circ} \mathrm{C}$. After the gas evolution stopped $(24 \mathrm{~h})$, the solution was filtered, and all volatile components were completely evaporated in vacuo. In the residue, $2.4 \mathrm{~g}$ of colloidal platinum powder was obtained in the form of a black, air-sensitive powder. The content of reactive $\mathrm{Al}-\mathrm{CH}_{3}$ groups in the powder was determined by protonolysis. For that purpose, the colloid was dissolved in toluene, and $30 \%$ acetic acid in toluene was added. The amount of gas evolved during the addition was measured and analyzed by GC/MS. A typical protonolysis experiment of a Pt colloid is described in ref 16.

Formation of Pt Nanoparticle Networks. The Pt colloid $(0.5 \mathrm{~g})$ was dissol ved in $500 \mathrm{~mL}$ of dry tetrahydrofuran (THF). The respective spacer molecule was dissolved in $200 \mathrm{~mL}$ of THF and added dropwise to the colloidal solution. The amount of $\mathrm{OH}$ groups added was equal to the amount of $\mathrm{Al}-\mathrm{CH}_{3}$ groups in the colloid. The mixture was stirred overnight at ambient temperature. The colloidal network precipitated and was filtered and washed with THF to remove excess spacer molecules.

Scattering Experiments. The ASAXS measurements were performed at the J USIFA beamline ${ }^{24}$ at the DORIS III storage ring of the Deutsches Elektronen Synchrotron (DESY, Hamburg, Germany).

\section{Results and Discussion}

Synthesis of Pt Nanoparticles Having Reactive Aluminum Organic Protecting Groups. The platinum particles are formed by the reaction of platinum(II) acetylacetonate $\left[\mathrm{Pt}(\mathrm{acac})_{2}\right]$ with trimethylaluminum at $60^{\circ} \mathrm{C}$ in an argon atmosphere and absolute dry toluene as the solvent. A scheme of the reduction process is shown in Figure 1 in which the known features of the reaction and the resulting particles are summarized. The progress of the reduction can be observed by measuring the amount of gas evolving during the reaction. The gas consists mainly of methane (>90 mol $\%$ ), ethylene, and ethane. In situ NMR and mass spectrometric investigations on the colloid synthesis indicate the formation of a diplatinum tetramethyl complex with two bridging aluminum methyl groups (see Figure 1) and dimethylal uminum acetylacetonate in early stages of the reaction. The platinum particles result from the decomposition of the diplatinum complex. (A detailed study on the mechanism of colloid formation will be published together with additional investigations). However, in the colloid synthesis, an excess of aluminum alkyl (Pt/Al ratio of 1:4) is used to ensure the complete reduction of the Pt salt. This excess is needed because of side reactions taking place between trimethylaluminum and dimethylaluminum acetylacetonate, which lead to more complex organometallic structures.

The platinum colloid can be isolated by evaporating the solvent and all residual volatile material in vacuo. The resulting black, air-sensitive colloid powder can be redispersed in aprotic organic solvents such as toluene or THF. Elemental analysis reveals a Pt/AI ratio of approximately 1:2. The structure of the colloidal particles and the surrounding shell is still under investigation. The following characterizations show that the schematic model shown in Figure 1 is a good approach to the real structure of the colloidal particles.

Transmission Electron Microscopy I nvestigations. Figure 2a shows a TEM image of a dilute dispersion of the colloid powder in THF dropped onto a carbon-coated copper grid (400 mesh/in.). As the image shows, the powder consists of spherical particles having a mean diameter of $1.2 \mathrm{~nm}$. The corresponding histogram (F igure $2 \mathrm{~b}$ ) reveals that the particle size distribution is rather narrow and exhibits the features of a log-normal distribution. The investigation of the particle structure with HRTEM shows that most of the particles appear to be amorphous. Nevertheless, some of the larger particles ( $\geq 2 \mathrm{~nm}$ ) exhi bit crystalline structures that can be identified by their typical lattice fringes (Figure 3). Moreover, X-ray absorption near-edge structure (XANES) measurements on the colloid powder show that the particles are mainly reduced to the zero-val ent state. ${ }^{18}$

IR and NMR Spectroscopic I nvestigations. As al ready mentioned, the key feature that makes the particles suitable for further reactions at the colloidal protecting shell is the presence of aluminum alkyl groups. These

(18) Vad, T.; Haubold, H.-G.; Waldöfner, N.; Bönnemann, H. J . Appl. Cryst., manuscript submitted. 
(a)

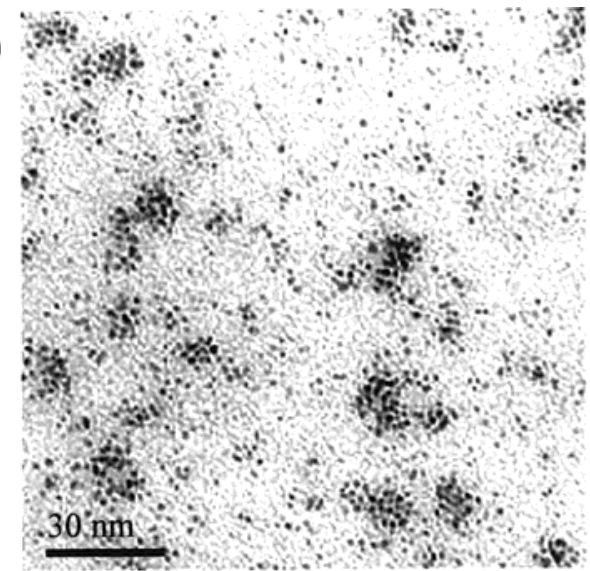

(b)

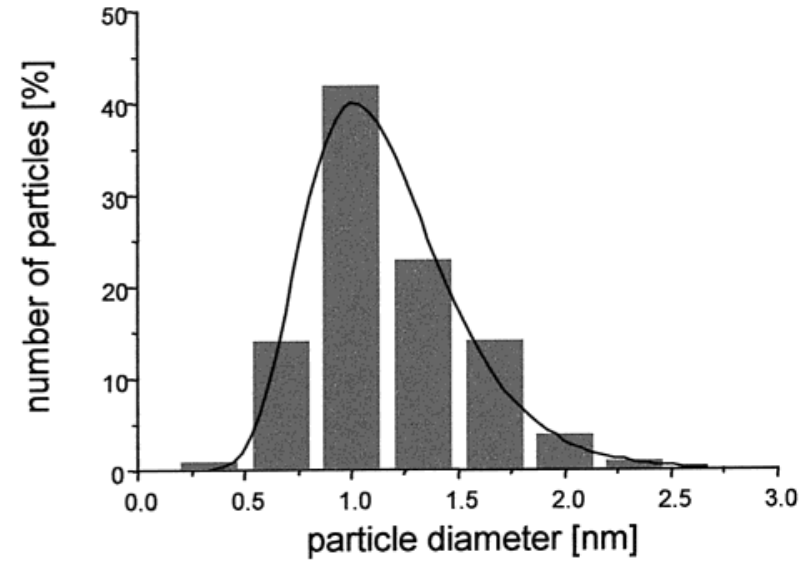

Figure 2. TEM analysis of aluminum-organic-stabilized Pt nanoparticles: (a) TEM image, (b) corresponding particle size distribution.
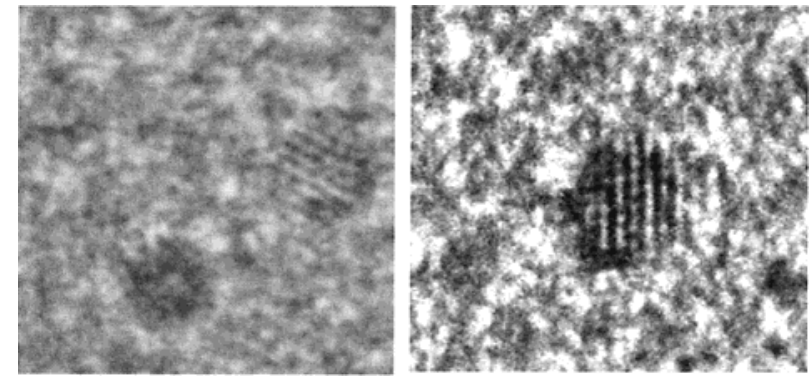

Figure 3. HRTEM images of larger Pt particles showing lattice fringes.

(a)

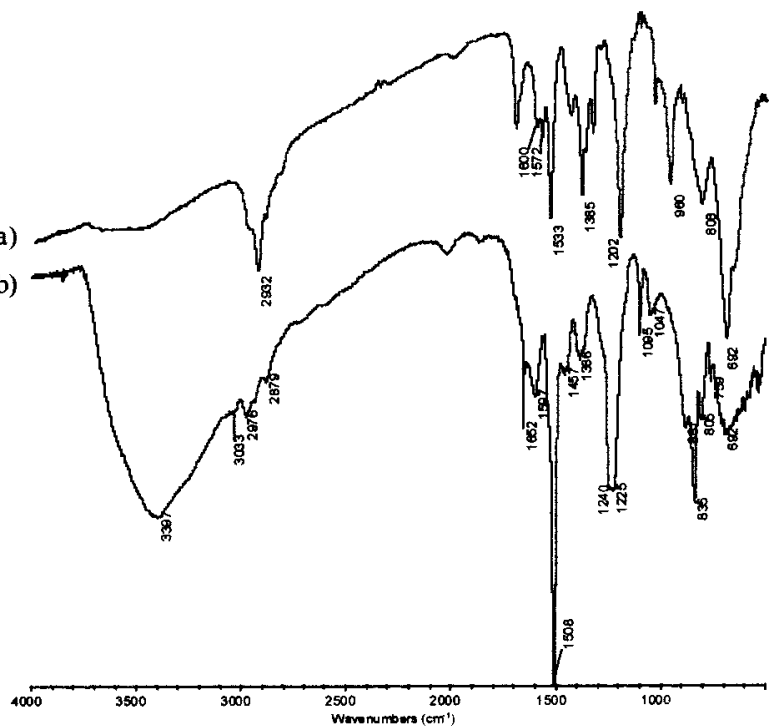

Figure 4. IR analysis of the colloid powders: (a) IR spectrum of the free aluminum-organic-stabilized Pt colloid, (b) IR spectrum of the colloid after cross-linking with hydroquinone.

highly reactive $\mathrm{Al}-\mathrm{CH}_{3}$ bonds can be easily identified by IR spectroscopy. Figure 4a shows an IR spectrum of the colloidal powder containing the free (not crosslinked) Pt particles. At $1202 \mathrm{~cm}^{-1}$, we see a $\mathrm{C}-\mathrm{H}$ deformation mode that corresponds to the methyl group bonded to the aluminum. ${ }^{19}$ Another piece of evidence is the presence of the $\mathrm{Al}-\mathrm{C}$ valency vibration mode at 692 $\mathrm{cm}^{-1}$. In addition, IR spectroscopy reveals a shift of the

(19) Hoffmann, E. G. Ber. Bunsen-Ges. Phys. Chem. 1960, 64, 4 (5), 616-631.

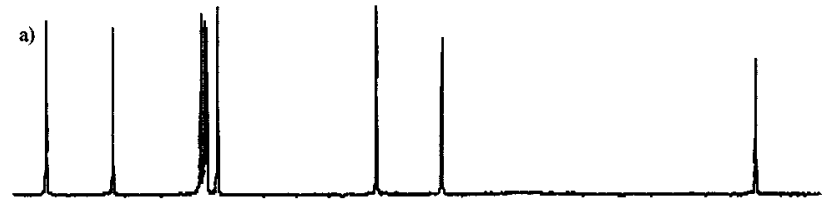

b)

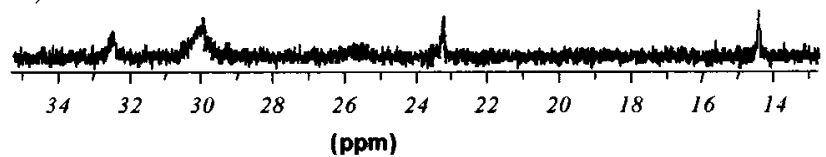

Figure 5. Comparison of ${ }^{13} \mathrm{C}$ NMR spectra of (a) free decanoic acid and (b) a Pt colloid modified with decanoic acid .

metal-sensitive vibration modes of the acetylacetonate group, 20 which indicates that a ligand exchange from Pt to Al occurred. The vibration at $1600 \mathrm{~cm}^{-1}$ is similar to that of pure dimethylaluminum acetylacetonate (1590 $\mathrm{cm}^{-1}$ ). On the other hand, the mode at $1572 \mathrm{~cm}^{-1}$ is closer to that of pure platinum(II) acetylacetonate (1560 $\mathrm{cm}^{-1}$ ), which might be due to the presence of acac groups coordinated to $\mathrm{Pt}$ at the particle surface. In addition to these observations, which lead to the particle model shown in Figure 1, the spectral features assume a more complex structure of the colloidal shell, which is not currently completely understood.

Because of the colloidal nature of the organometallic protecting shell, the NMR resonances are broadened and cannot be analyzed by standard NMR methods. Figure $5 \mathrm{~b}$ shows the ${ }^{13} \mathrm{C}$ NMR spectrum of an aluminumorganic-stabilized Pt colloid after addition of decanoic acid. The addition of the acid yiel ds a modified ${ }^{17}$ colloid in which the acid is bonded to the aluminum of the protecting shell. A comparison to the spectrum of free decanoic acid reveals that the signals of the acid in the colloid are broadened and some resonances even disappear. This confirms that the modifier is attached to the particle shell.

Cross-linking of Aluminum-Organic-Stabilized Pt Particles with Bifunctional Alcohols. The reactive nature of the colloidal shell allows protonolytic reactions at the surface of the colloidal particles to occur. This can easily be demonstrated by adding a solution of monofunctional alcohols or dicarboxylic acids to the

(20) Nakamoto, K.; McCarthy, P. J .; Ruby, A.; Martell, A. E. J . Am. Chem. Soc. 1961, 83, 1066-1069. 

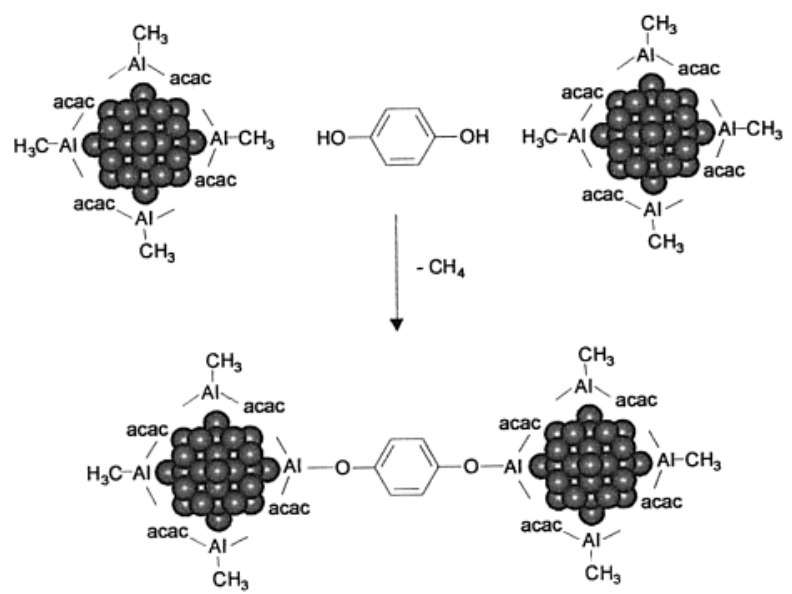

Figure 6. Principle of the protonolytic cross-linking mechanism.

Table 1. Structural Data on the Colloidal Powders and Spacers as Determined from Molecular Modeling ${ }^{21}$ and Scattering Curves

\begin{tabular}{lccc}
\hline \multicolumn{1}{c}{ sample } & $\begin{array}{c}\text { O-O distance } \\
\text { of the spacer } \\
\text { (molecular } \\
\text { modeling } \\
(\AA)\end{array}$ & $\begin{array}{c}\text { Q value } \\
\text { at peak } \\
\text { maximum } \\
(1 / \AA)\end{array}$ & $\begin{array}{c}\text { typical } \\
\text { distance } \\
\mathrm{d}=2 \pi / \mathrm{Q}_{\max } \\
(\AA)\end{array}$ \\
\hline free Pt colloid & - & 0.314 & 20.01 \\
Pt/hydroquinone & 5.6 & 0.250 & 25.13 \\
Pt/4,4'-dihydroxybiphenyl & 9.9 & 0.203 & 30.95 \\
Pt/1,10-decanediol & $13.8^{\mathrm{a}}$ & 0.229 & 27.44
\end{tabular}

a Maximum length calculated for zigzag configuration.

coll oi dal dispersion. The amount of gas evolving during this process is a measure of the number of reacted $\mathrm{Al}-$ $\mathrm{CH}_{3}$ groups in the colloid. Using this modification pathway, it is possible to alter the dispersive properties of the colloids by varying the chemical nature of the modifier. The approach presented in this paper is based on the protonolytic reaction with bifunctional alcohols. Because of the presence of a second $\mathrm{OH}$ group in these diols, a connection of particles can be brought about as depicted in Figure 6. As the particles are statistically covered with reactive $\mathrm{Al}-\mathrm{CH}_{3}$ groups, this reaction leads to the formation of a 3-D cross-linked nanoparticle network. In contrast to the modification that yields redispersible colloids, this reaction results in the precipitation of an insoluble powder.

The structure of the bifunctional alcohols used as spacers plays an important role during the formation of the inorganic/organic network. The fabrication of a regularly arranged colloidal network with chemically tailored properties requires control over the interparticle distances. Starting from a colloidal particle with a given diameter and thickness of the surrounding shell, control over the interparticle distance can be achieved by inserting spacers with different lengths. As a starting point, we chose the different spacer systems shown in Table 1. Hydroquinoneand 4,4'-dihydroxybi phenyl have more rigid and rodlike structures. As a comparison, we also used the more flexible 1,10-decandiol whose length depends more or less on the conformational structure. The spacer lengths were calculated using molecular modeling ${ }^{21}$ and are in the size range of the diameter of the Pt particles.

(21) SYBYL, v.6.5.1., Tripos Assoc., Inc.: St. Louis, MO.
TEM and IR Investigations of Network Formation. In Figure 7, the results from TEM investigations of the formation of $\mathrm{Pt} /$ (ethylene glycol) hybrids are shown. The TEM images reflect the colloidal dispersion during the addition of different amounts of ethylene glycol. At low amounts of ethylene glycol, free particles and oligomeric structures can be observed. U pon subsequent addition of more ethylene glycol, these oligomers grow to higher polymeric structures. Finally, after all $\mathrm{Al}-\mathrm{CH}_{3}$ groups are protonolyzed, large micron-sized arrays of Pt particles have been formed. As the TEM images show, these arrays consist of nonagglomerated particles that are separated. Indeed, the main part of the particles is not visible in the TEM images because of the high degree of cross-linking.

The incorporation of aromatic spacers in the hybrid material can be followed by IR spectroscopy. Figure 4b shows the IR spectrum of a hydroquinone cross-linked Pt colloid. The presence of the aromatic ring can be identified by the $\mathrm{C}=\mathrm{C}$ vibrations at $1508 \mathrm{~cm}^{-1}$. Moreover, the spectrum shows that some vibrational modes typical of free hydroquinone are absent or shifted to different wavenumbers. For example, the aryl-O vibrational mode is shifted to higher wavenumbers (1225, $\left.1240 \mathrm{~cm}^{-1}\right)$ in comparison to that of the free hydroquinone $\left(\sim 1200 \mathrm{~cm}^{-1}\right)$. This reflects the conversion of the $\mathrm{R}-\mathrm{OH}$ groups to $\mathrm{R}-\mathrm{O}-\mathrm{Al}$ during the protonolytic reaction, which establishes the linkage between the particles. Furthermore, we see that the $\mathrm{Al}-\mathrm{CH}_{3}$ groups of the free colloid have almost quantitatively disappeared, which demonstrates the completeness of the protonolytic reaction. The fact that we still see the $-\mathrm{OH}$ vibrations from the hydroquinone is due to spacer molecules that are linked at only one side to the aluminum and still bear the $\mathrm{OH}$ group at the opposite end. Thus, not all spacers contribute to the cross-linking of the particles.

Anomalous Small-Angle X-ray Scattering. We applied anomalous small-angle X-ray scattering (ASAXS) to study the structural properties of the Pt particles without contributions of the organic part or porelike structures. To separate the scattering contributions of the platinum particles from those of the organic part, a contrast variation at the Pt(LIII) edge was applied. 18,22 In Figure 8, the scattering curves of the free alumi numorganic-stabilized Pt colloid at X-ray energies of E $1=$ $11545 \mathrm{eV}$ and E2 = $10976 \mathrm{eV}$ are shown. The curves show the scattering intensity as a function of the wave vector $Q[Q=(4 \pi / \lambda) \sin \theta]$. To extract the part of the scattering originating from Pt structures, these curves were subtracted from each other. The resulting curve $(E 2-E 1)$ reflects the scattering behavior of the $\mathrm{Pt}$ particles. The curve shows a hump at about $\mathrm{Q}=0.3$ $\AA^{-1}$ and reveals a correlation. Through this correlation effect, it is possible to determine the mean interparticle distances in the colloid.

The contrast variation for the Pt particle networks with organic spacer molecules was made similar to that of the free Pt colloid. In Figure 9, the correlation peaks of the two phenyl-cross-linked networks are compared to that of the free colloid (linear scaling). In the networks as well as in the free colloid, correlation effects

(22) Haubold, H.-G.; Wang, X. H.; Goerig, G.; Schilling, W. J . Appl. Cryst. 1997, 30, 653-658. 

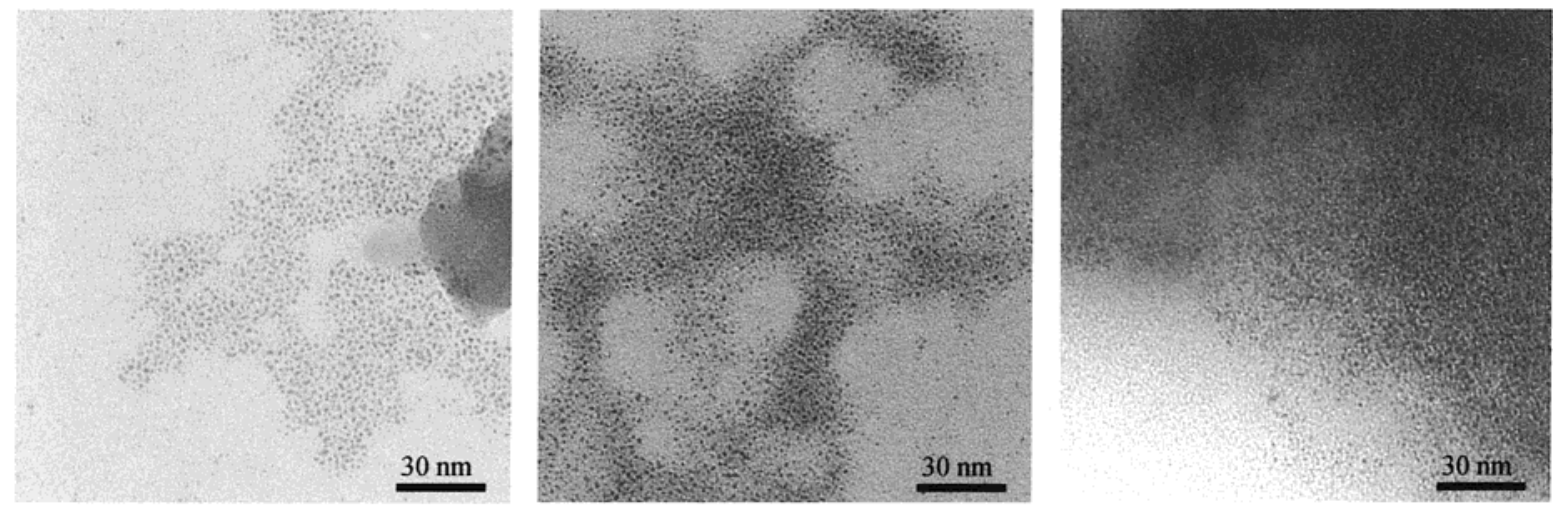

increasing amount of ethylene glycol added

Figure 7. TEM images of the nanoparticle network formation during cross-linking with ethylene glycol.

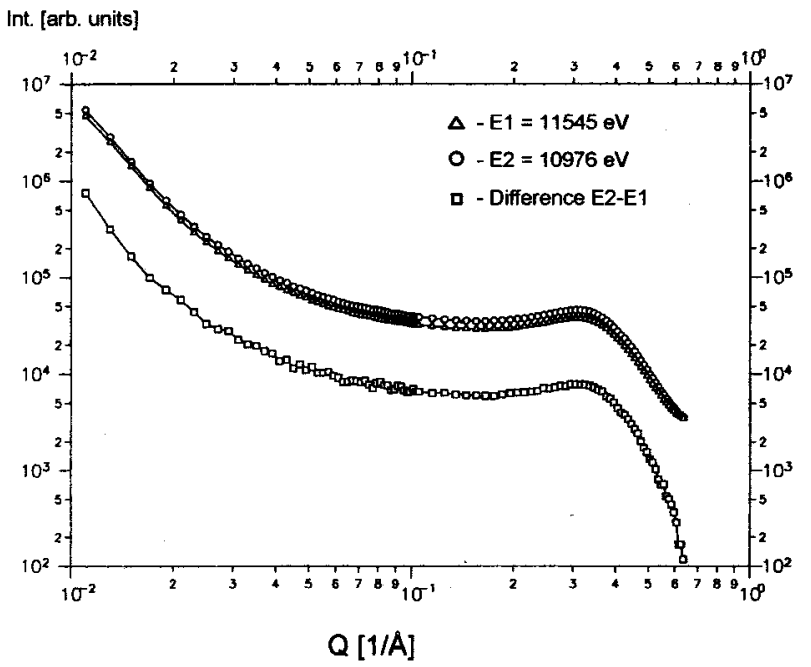

Figure 8. ASAXS results for the free aluminum-organicstabilized Pt colloid powder (contrast-variation plot).

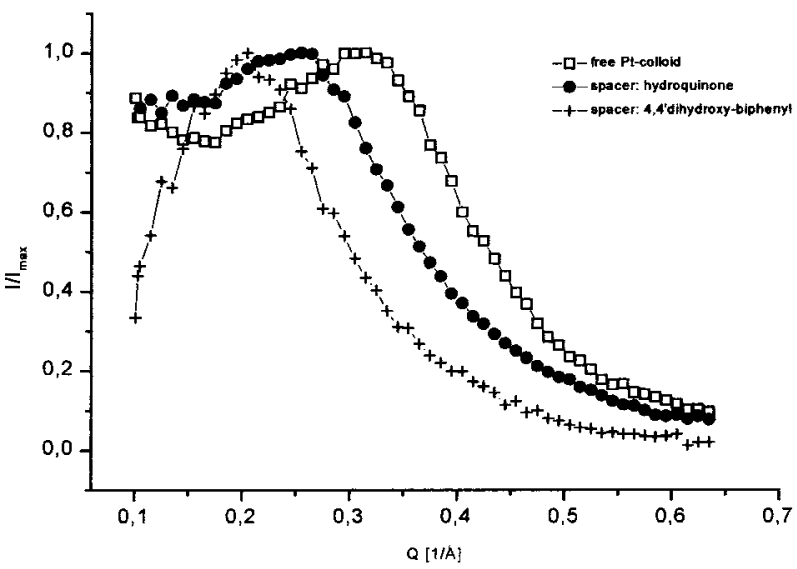

Figure 9. Comparison of the correlation peaks for cross-linked and non-cross-linked colloids. (The scattering curves are normalized to the maximum intensity of the correlation peak.)

between the particles can be observed. The free colloid shows a correlation peak with a maximum at a $\mathrm{Q}$ value of approximately $0.31 \AA^{-1}$ (spacing $d=2 \pi / Q \approx 20 \AA$ ). The most obvious result is a shift of the correlation peak to lower $\mathrm{Q}$ values and thus to higher interparticle distances after insertion of the spacer molecules. The peak maximum is shifted to $0.25 \AA^{-1}$ in the hydro-
Table 2. Average Model Parameters $R_{0}$ (Particle Radius), $D_{0}$ (Distance between the Particle Surfaces), and $D_{p s}$ (Thickness of the Protecting Shell)

\begin{tabular}{lccc}
\hline \multicolumn{1}{c}{ sample } & $\mathrm{R}_{0}(\AA)$ & $\mathrm{D}_{0}(\AA)$ & $\mathrm{D}_{0}-2 \mathrm{D}_{\mathrm{ps}}(\AA)$ \\
\hline free Pt colloid & 5.5 & $3.7\left(\sim 2 \mathrm{D}_{\mathrm{ps}}\right)$ & - \\
Pt/hydroquinone & 5.3 & 9.8 & 6.1 \\
Pt/4,4'-dihydroxybiphenyl & 6.2 & 12.3 & 8.6
\end{tabular}

quinone network and to $0.20 \AA^{-1}$ in the case of the $4,4^{\prime}$ dihydroxybi phenyl network. This corresponds to spacings $(2 \pi / \mathrm{Q})$ of $25 \AA$ for the Pt/hydroquinone network and $31 \AA$ for the Pt/bi phenyl network (see Table 1).

These spacings, however, reflect the interparticle distances only approximately, as the Q dependence of the particle form factor has to be taken into account for a more precise determination. To obtain the exact particle sizes and distances, a modified fit model based on the work of A. Vrij 18,23 was applied. As Table 2 shows, the results for the (most frequently occurring) radii are in excellent agreement with those obtained by electron microscopy. Moreover, the averaged distance $D_{0}$ between the particle surfaces can be calculated. The different $D_{0}$ values of the three samples are found to be consistent with the different types of spacer molecules used for the interconnection of the metal particles. For the free Pt colloid, the refinement yields a $D_{0}$ value of approximately $4 \AA$. This value should correspond roughly to twice the thickness, $D_{p s}$, of the aluminum-organic protecting shell that surrounds each of the particles. However, the thickness of $2 \AA$ is quite small, which implies that part of the aluminum-organic material forms some kind of alloy together with the Pt atoms at the particle surface. For the cross-linked networks, $\mathrm{D}_{0}$ values of $9.8 \AA$ (Pt/hydroquinone) and $12.3 \AA$ (Pt/4,4'dihydroxy-bi phenyl) were obtained. The differences $D_{0}$ $-2 D_{p s}$ of $6.1 \AA$ for the Pt/hydroquinone and $8.6 \AA$ for the Pt/4,4'-dihydroxybiphenyl hybrid are consistent with the theoretical spacer lengths calculated by molecular modeling (see Table 1).

These measurements demonstrate that it is possible to control the interparticle distance in the colloidal

(23) Vrij, A. J . Chem. Phys. 1979, 71, 3267-3270.

(24) Haubold, H.-G.; Gruenhagen, K.; Wagener, M.; J ungbluth, H.; Heer, H.; Pfeil, A.; Brandenburg, H.; Moeller, R.; Matzerath, J .; Hiller, P.; Halling, H. Rev. Sci. Instrum. 1989, 60, 1943-1946. 
network by cross-linking with different spacer molecules.

\section{Conclusions}

We have shown that aluminum-organic-stabilized Pt colloids can be used as a precursor for the arrangement of nanoparticles in 3-D networks. Because of their highly reactive protecting shell, these particles allow for protonolytic chemical reactions at the particle surface. For network production, the highly reactive colloidal particles can be cross-linked with bifunctional alcohols. As shown by a combination of TEM, IR and ASAXS analyses, the Pt particles are incorporated into a 3-D network without changing their size. Furthermore, the scattering results show that the interparticle distance increases after incorporation of the spacer molecules.
The distances determined are in good agreement with the spacer lengths calculated by molecular modeling. Thus, this method allows for control over the interparticle distance by using spacers of different lengths. Furthermore, if one succeeds in preparing more monodisperse reactive stabilized nanoparticles, the formation of highly ordered networks should be possible.

Acknowledgment. Financial support from the Deutsche Forschungsgemeinschaft (DFG) within the program Halbleiter und Metallcluster als Bausteine für organisierte Strukturen is gratefully acknowledged. The authors also thank $\mathrm{Dr}$. B. Tesche (MPI für Kohlenforschung) for the TEM studies cited in this work.

CM 0111837 